\title{
On Shear-Driven Ventilation of Snow
}

\author{
Andrew Clifton • Costantino Manes • \\ Jean-Daniel Rüedi • Michele Guala • Michael Lehning
}

Received: 13 April 2007 / Accepted: 4 October 2007 / Published online: 8 November 2007

(C) Springer Science+Business Media B.V. 2007

\begin{abstract}
A series of experiments have been made in a wind tunnel to investigate the ventilation of snow by shear. We argue that the zero-plane displacement can be used as a convenient indicator of ventilation, and that this can be obtained from measurements of mean velocity profiles in conditions of zero pressure gradient. Measurements made over a natural snow surface show a zero-plane displacement depth of less than $5 \mathrm{~mm}$, but practical considerations preclude extensive use of snow for these measurements. Instead, the influence of permeability is investigated using reticulated foams in place of snow. We demonstrate that the foam and snow have similar structure and flow-relevant properties. Although the surface of the foam is flat, the roughness lengths increase by two orders of magnitude as the permeability increases from $6 \times 10^{-9}$ to $160 \times 10^{-9} \mathrm{~m}^{2}$. The zero-plane displacement for the least permeable foams is effectively zero, but more than $15 \mathrm{~mm}$ for the most permeable foams. Our data compare well to the few studies available in the literature. By analogy to conditions over snow surfaces, we suggest that shear-driven ventilation of snow is therefore limited to the upper few millimetres of snow surfaces.
\end{abstract}

Keywords Boundary-layer meteorology $\cdot$ High latitudes $\cdot$ Porous media · Snow $\cdot$ Ventilation $\cdot$ Zero-plane displacement

\section{Introduction}

Energy and mass exchange processes between snow covered surfaces and the atmosphere play an important role in various fields of meteorology and climatology. Research has attempted to understand the mechanisms by which chemical species are incorporated into the snow in order

A. Clifton $(\varangle) \cdot$ C. Manes $\cdot$ J.-D. Rüedi $\cdot$ M. Guala $\cdot$ M. Lehning

Swiss Federal Institute for Snow and Avalanche Research SLF, Flüelastrasse 11, Davos Dorf 7260 Switzerland

e-mail: clifton@slf.ch

A. Clifton

University of Northern British Columbia, Prince George, BC, Canada 
Fig. 1 Mean velocity profiles over and within a porous surface. Profiles are shown with and without a pressure gradient in the streamwise direction $(d P / d x) . z$ is the vertical co-ordinate, $h$ is the depth of the porous media and $u(0)$ is the slip velocity

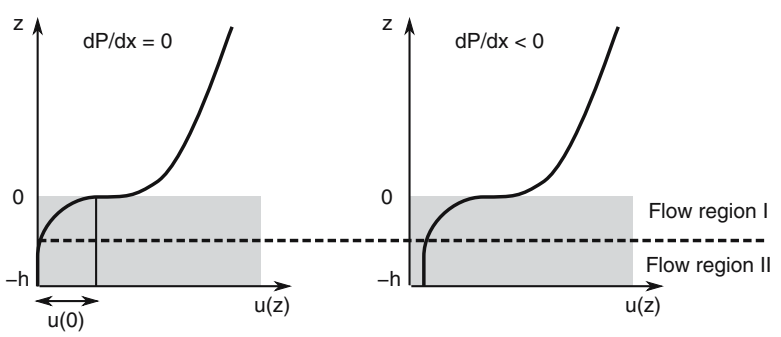

to reconstruct the history of climate from ice core samples taken in Polar and high-latitude terrain. Furthermore, the uptake of chemical species by the snow and the photochemical and physicochemical reactions within the snow pack significantly affects atmospheric chemistry (Albert and Schultz 2002). Mass exchange processes also need to be understood to predict water vapour exchange, which is very important for hydrological and snow cover models.

Atmospheric flow over a snow covered surface is a complicated process as it involves flow interacting with a porous medium. This flow can even be two-phase, when drift occurs. The current knowledge on this topic is still very limited. As it is a porous medium, snow experiences interstitial air movements, which give rise to so-called ventilation. It has been argued by many authors that ventilation significantly affects mass and energy exchange processes (Albert and Schultz 2002; Albert et al. 2002). Several studies have been carried out to clarify the mechanisms by which air movement is induced within the snow (Colbeck 1989; Sturm and Johnson 1991; Sokratov and Sato 2000, 2001). Waddington and Cunningham (1996) point out that ventilation inside the snow pack can be induced by pressure gradients, temperature gradients and, at smaller scales, by turbulence in the atmospheric boundary layer above the snow pack. This paper focuses on this last mechanism and aims to clarify how a turbulent boundary layer can induce air movements within a snow pack. We consider the case of a flat and uniform snow cover below a turbulent boundary layer, corresponding to conditions found in high latitudes and Greenland, for example.

Recent studies on turbulent boundary layers over and inside porous media by Breugem et al. (2006) and Manes (2006) have shown that the flow within a flat porous surface can be divided into two flow regions (see Fig. 1). These are (I) an upper region where significant turbulent momentum transfer induces a slip velocity and large gradients of mean velocities occur, and (II) a lower region where shear disappears but velocity fluctuations still persist. These fluctuations are induced by the turbulent boundary layer, which imposes pressure fluctuations within the porous surface. This turbulence is said to be 'inactive' in the sense that it is not able to induce momentum transfer, contrary to the 'active' turbulence in the upper layer.

Mean velocities in region (II) (where shear is the driving force for ventilation) decay to zero in the case of a zero pressure-gradient boundary layer. In a boundary layer with non-negligible horizontal pressure gradient, mean velocities asymptotically match a constant mean value representative of a Darcy-type flow (Fig. 1). In Darcy-type flow, the rate of throughflow is inversely proportional to the permeability, where the permeability of a porous medium represents the capacity of that medium to absorb momentum from a fluid moving within it.

The novelty of the work published here is in establishing the extent to which shear can penetrate within a porous snow pack and hence cause shear driven ventilation. To the authors' knowledge, previous studies on this topic have been unable to definitively distinguish between ventilation that was induced by shear or by horizontal pressure gradients, either from synoptic forcing (Albert and Schultz 2002) or boundary-layer growth (Sokratov and Sato 2000). When modelling mass exchange processes this is a crucial question. If shear penetration is 
significant, by analogy one can infer that turbulent scalar fluxes are also significant within the snow pack and these fluxes strongly influence processes of mass release or uptake. One could also hypothesise that inactive turbulence within region (II) might play a role in influencing snow metamorphism (e.g., enhancing air mixing within the snow pack) but this type of study is beyond the scope of this paper and the focus is only on shear penetration.

Assessing shear penetration within a porous medium under a turbulent boundary layer is not an easy task. To have exact estimates one should measure either turbulent fluxes or mean velocities within the porous medium and determine at which elevation their gradient and hence the shear effect disappears. However, measuring turbulent flow velocities within the small voids of a porous medium is very difficult and reliable experimental techniques are not yet available. Here, we suggest a way of bypassing this problem and evaluate shear penetration from standard mean velocity measurements within the boundary layer that are much easier to perform. The analysis is based on Jackson (1981), who shows how shear penetration is related to the zero-plane displacement of the wall.

Experiments were carried out in a wind tunnel using snow or porous reticulated foams. Foam is preferred to snow since it represents a more controlled system and shear penetration can be estimated for different values of porosity and permeability. Other influences such as drift, roughness texture or vertical heterogeneity of snow due to settling effects are avoided. A variety of foams were chosen in order to provide a wide range of permeabilities and porosities, including the typical values encountered for real snow packs in order to be able to extend the results to real world problems.

Details on the experimental methodology and results are shown after introducing the theoretical background used to estimate shear penetration from mean velocity profiles.

\section{Theoretical Background}

It is well known that mean velocity profiles in the near-wall region of a turbulent boundary layer over rough walls can be expressed by the logarithmic profile. This gives the velocity at a height above the surface, $u(z)$ as

$$
u(z)=\left(\frac{u_{*}}{\kappa}\right) \ln \left(\frac{z+d}{z_{0}}\right)
$$

where $d$ is the zero-plane displacement, $z_{0}$ is the roughness length, $u_{*}$ is the friction velocity and $\kappa$ is the von Kármán constant, taken here as 0.41 .

Jackson (1981) noted that in a zero pressure gradient turbulent boundary layer over a rough surface, $d$ is the depth at which the mean drag on the surface appears to act. Expressed formally, this gives;

$$
d=\frac{\int_{-h}^{0} z \mathcal{F}(z) d z}{\int_{-h}^{0} \mathcal{F}(z) d z}
$$

where $\mathcal{F}(z) d z$ is the drag force acting within the porous medium at a given height over an element of size $d z$, and $h$ is the total depth of the medium. This force acts on the matrix (the solid part) of the material. In the case of a zero pressure-gradient boundary layer in neutral conditions, the mean drag force at a height within the porous medium is equal to the local shear stress gradient, given by

$$
\mathcal{F}(z)=\frac{d \tau}{d z}
$$

and Eq. 2 becomes 


$$
d=\frac{\int_{-h}^{0} z \frac{d \tau}{d z} d z}{\int_{-h}^{0} \frac{d \tau}{d z} d z}
$$

where $\tau$ is the local shear stress. In a fully turbulent boundary layer, it is reasonable to assume that this shear stress is due to a downwards turbulent momentum flux. It was therefore suggested that $d$ is a length scale related to the shear penetration of the flow within the roughness elements. Jackson verified this hypothesis with a variety of rough surfaces, and observed that $d$ was mainly a function of surface geometry. Earlier experiments over plant canopies by Thom (1971) substantiate Jackson's findings, but Thom pointed out that $d$ was also a function of flow energy, i.e., $u_{*}{ }^{2}$. In fact it was observed that for an increase of $u_{*}$, the zero plane position was shifted downwards deeper within the roughness elements. The dependence of $d$ on $u_{*}$ and roughness geometry was also observed in open channel flows by Nikora et al. (2002).

In the experiments presented herein, $d, u_{*}$ and $z_{0}$ were estimated by fitting mean velocities to Eq. 1 using the method of Clauser (1954). The vertical origin for $z$ was chosen at the top of the foam. As $d$ tends to zero, shear penetration becomes smaller, and at the limit, when $d=0$, the surface approaches the behaviour of a solid wall, and flow hardly penetrates the porous medium.

\section{Materials}

Snow is formed when water vapour condenses, freezes and forms agglomerates. These agglomerates (flakes) can be single or multiple crystals, but are essentially discrete granules as they pass through the atmospheric boundary layer. When snow flakes reach a snow surface, they rapidly bond to snow already present, forming a porous and permeable threedimensional ice matrix. A snow surface can therefore be considered to be a foam of ice. Variations in cloud temperature and humidity result in different forms of snow being deposited on the surface. Further changes in snow forms are caused by heat fluxes in the snow pack, and erosion and deposition by wind (wind packing), which are then covered by fresh falls of snow. These processes cause clearly identifiable layers to develop in the snow, each of which has different morphology. The permeability of the snow then also changes throughout the snow pack, depending on snow microstructure. Measured values of porosity, defined as the percentage of the snow that is void, and permeability for various snow types, taken from the literature, are given in Table 1.

Table 1 Porosity and permeability $(k)$ of different snow types from the literature

\begin{tabular}{|c|c|c|c|}
\hline Material & Porosity (\%) & $k\left(\times 10^{-9} \mathrm{~m}^{2}\right)$ & Source \\
\hline New snow, wind blown & $83-85$ & $2.6-3.1$ & $\begin{array}{l}\text { Seasonal snow cover (Sommerfeld and } \\
\text { Rocchio 1993) }\end{array}$ \\
\hline Wind-packed snow & 77 & 1 & $\begin{array}{l}\text { Siple Dome, Antarctica (Albert et al. } \\
\text { 2000) }\end{array}$ \\
\hline Firn & - & 28 & \\
\hline Melt-freeze surface & 63 & $6-14$ & $\begin{array}{l}\text { Seasonal snow cover (Albert and Perron } \\
\text { 2000) }\end{array}$ \\
\hline Melt-freeze crystals & 62 & $5-11$ & \\
\hline Ice layer & - & $0.03-1.4$ & \\
\hline
\end{tabular}


Illustrative images of the microstructure of fresh snow and of one of the polyester foams used in this series of experiments are shown in Fig. 2. These images were obtained by X-ray tomography (Schneebeli and Sokratov 2004). As can be seen from the figure, the snow and foam are qualitatively similar in that the media are highly porous and the structure is composed of long, thin structures that connect at nodes. However, the snow is more complex and less regular than the artificial foam. The geometric form of the material can be characterised using the specific surface area, SSA, which is defined as the surface area of the medium, per volume, and has dimensions $\mathrm{m}^{-1}$. The surface area of the solid matrix can be obtained by reconstruction of the 3D surface from the tomographic data, which also gives the porosity. The SSA and porosity $\phi$ can then be used to establish the diameter of a particle with the same SSA; this is denoted as the equivalent particle diameter $d_{p}$, which from geometry is given by $d_{p}=6 \times(1-\phi) / S S A$ (Breugem et al. 2006). Foam permeability $k$ was measured by T. Neumann (U. Vermont) and Z. Courville (Dartmouth College) using a through-flow permeameter (Albert and Perron 2000). The SSAs of the fresh snow in Fig. 2a, and the SSA and permeability of the foam used in the experiments, are detailed in Table 2.

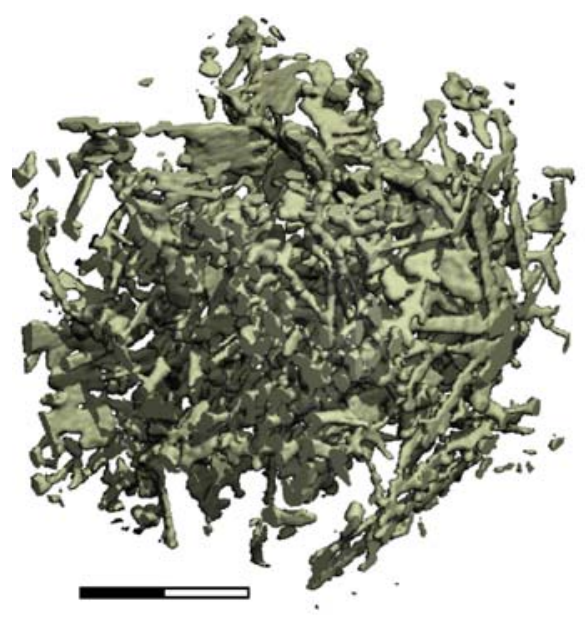

(a) Snow

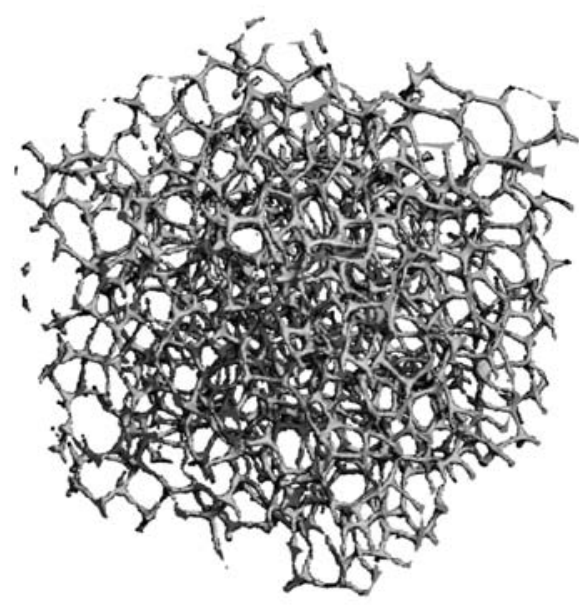

(b) Foam

Fig. 2 Three-dimensional reconstruction of the microstructure of foams. (a) New snow, density $54 \mathrm{~kg} \mathrm{~m}^{-3}$. The scale bar is $1 \mathrm{~mm}$. (b) Regicell 60 reticulated foam. Material structure was imaged using an X-ray tomograph, and the scale is identical for both images. Images courtesy of M. Schneebeli, SLF

Table 2 Characteristics of snow and foam

\begin{tabular}{lllll}
\hline Material & Porosity $(\%)$ & $\ell_{\text {pore }}(\mathrm{mm})$ & $S S A\left(\mathrm{~mm}^{-1}\right)$ & $k\left(\mathrm{~m}^{2}\right)$ \\
\hline Fresh snow, Fig. 2a & 94.1 & 0.46 & 2.88 & $160 \times 10^{-9}$ \\
Regicell 10 foam & 96.4 & 3.9 & 0.37 & $39 \times 10^{-9}$ \\
Regicell 30 foam & 97.0 & 1.5 & 0.82 & $6 \times 10^{-9}$ \\
Regicell 60 foam & 98.0 & 0.5 & 1.70 & 6 \\
\hline
\end{tabular}

Data include porosity, mean pore size $\ell_{\text {pore }}$, the specific surface area SSA and permeability $k$. Snow data are illustrative. Foam names are trade names and given for completeness 


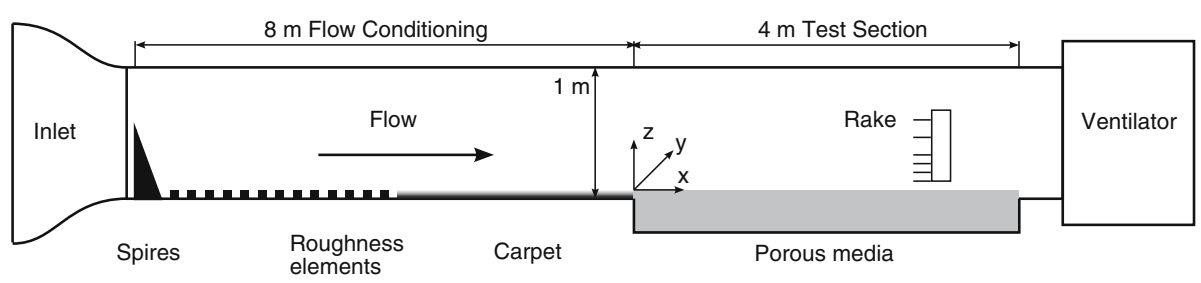

Fig. 3 Schematic view of the wind tunnel experiment

\section{Methods}

Mean velocities were measured in a boundary layer over several different snow surfaces and foams in the Swiss Federal Institute for Snow and Avalanche Research (SLF) boundary-layer wind tunnel. The SLF wind tunnel is designed to measure boundary-layer structure over variable depths of natural or artificial media. A sketch of the tunnel is shown in Fig. 3. The wind tunnel was originally designed to be used to measure snow transport under controlled conditions (Clifton et al. 2006).

The wind-tunnel roof line was adjusted so that the static pressure drop in the test section was as low as possible. Velocity profiles were measured over foam using a dynamic pressure rake after a $3 \mathrm{~m}$ fetch of medium. Velocity profiles over snow were measured using a hot-wire anemometer at about the same fetch. Results are described in detail in the following section, and, in general, show that a logarithmic boundary-layer velocity profile forms, and that the profile can be described using the friction velocity, the roughness length and the zero-plane displacement.

The velocity profiles in the logarithmic region were fitted using Eq. 1 after the method of Clauser (1954). First, we chose a range for the zero-plane displacement $d$ and optimised $u_{*}$ and $z_{0}$. A quality of fit was judged using the coefficient of determination $R^{2}$. The value of $d$ was then adjusted (either extended or refined) using the best result for $d$ from these results, and a new fit calculated. The search was iterated until the value of $d$ stabilised.

Sources of error in the calculation of $u_{*}, z_{0}$ and $d$ include those from the measurement devices, those from the vertical position and the fitting process itself. Using the errors given by the manufacturer for the equipment, and assuming $1 \mathrm{~mm}$ vertical accuracy, and then applying an error propagation technique (Moffat 1982), we estimate an uncertainty in $u_{*}$ of about $5 \%$ solely from the measurement chain. The uncertainty in $z_{0}$ we estimate to be about $40 \%$ for an actual $z_{0}$ of $1 \times 10^{-5} \mathrm{~m}$, decreasing to about $20 \%$ at an actual $z_{0}$ of $1 \times 10^{-3} \mathrm{~m}$. The pitot tubes may be affected by viscosity, turbulence and flow displacement (McKeon et al. 2003), but these influences are expected to be smaller than were assumed in the error propagation.

As the remaining sources of error cannot be estimated a priori, the data are validated by comparing mean velocity profiles measured over a smooth wall, with the classical law of the wall. A comparison of selected velocity profiles with the classical universal profile (Schlichting and Gersten 2003, p. 525) is presented in Fig. 4. Results are shown for a solid wall, snow and foams at the same nominal free-stream velocity. In the logarithmic region, $u^{+}=\frac{1}{\kappa} \ln \left(y^{+}\right)+C$, where $C$ is a constant. Our results show that each velocity profile has at least six points in the logarithmic region, where $u(z) / u_{*}$ is proportional to $\ln (z+d)$. Schlichting and Gersten give $C=5$ for a smooth wall, while Bhaganagar et al. (2004) give $C=5.5$. Raupach et al. (1991) shows that for a smooth wall, $z_{0}=\frac{v}{u_{*}} \exp (-\kappa C)$, which is plotted in Fig. 6 using the two values of $C$ given above. The standard deviation of $z_{0}$ measured over the solid smooth wall, from the expected value for a smooth floor, is $22 \%$ 


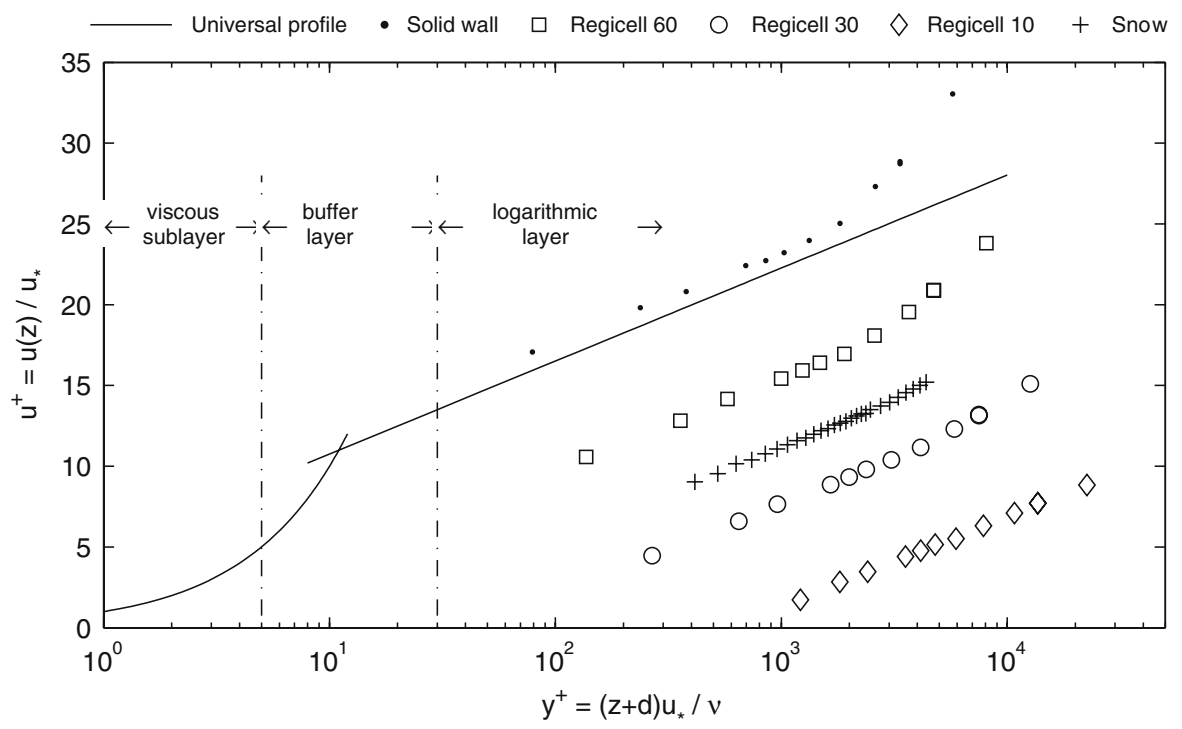

Fig. 4 Boundary layer profiles. Profiles were measured over a solid smooth wall, snow and foams (Regicell $n n$ ) in the SLF wind tunnel. The universal law of the wall for a smooth surface, where $u^{+}=\left(\frac{1}{\kappa}\right)$ $\ln \left(y^{+}\right)+5$ in the logarithmic region, (Schlichting and Gersten 2003, pp. 525-531), is plotted for comparison

if $C=5$, and $16 \%$ if $C=5.5$. The experiments reported here were made with upstream flow conditioning that was designed to mimic the atmospheric boundary layer for snow drift experiments (see Clifton et al. 2006). Because the wind-tunnel floor is then composed of patches with different roughness, an internal boundary layer forms over the material in the test section, and mean velocity data were taken only within that internal boundary layer. The good agreement with the universal profile shows that data have been used only from within the boundary layer that has developed over the surface of interest. Furthermore, as would be expected for a rougher surface, the log region increases in depth from the solid wall to the foams. These results demonstrate that the wind-tunnel and data processing routines are functioning correctly.

\section{Results}

\subsection{Snow Surfaces}

Examples of velocity profiles measured over a new snow surface are shown in Fig. 5. The permeability of the snow is unknown, but from Table 1 would be expected to be less than $5 \times 10^{-9} \mathrm{~m}^{2}$. The profiles were taken at two different free-stream velocities, giving two different $u_{*}$. No drift was observed during the measurements. The lower-speed profile shows no observable displacement, and $z_{0}$ was found to be $0.6 \mathrm{~mm}$ at $u_{*}=0.35 \mathrm{~m} \mathrm{~s}^{-1}$. At the higher speed, the velocity profile is lightly curved, and $u(z)$ does not scale directly with $\ln (z)$, indicating a slip velocity and thus a zero-plane displacement. The measured zero-plane displacement height in this case was $2.9 \mathrm{~mm}$, with $u_{*}=0.44 \mathrm{~m} \mathrm{~s}^{-1}$ and $z_{0}=0.35 \mathrm{~mm}$. The fitting routine converged in five iterations, giving a final $R^{2}>0.999$ between measurements 


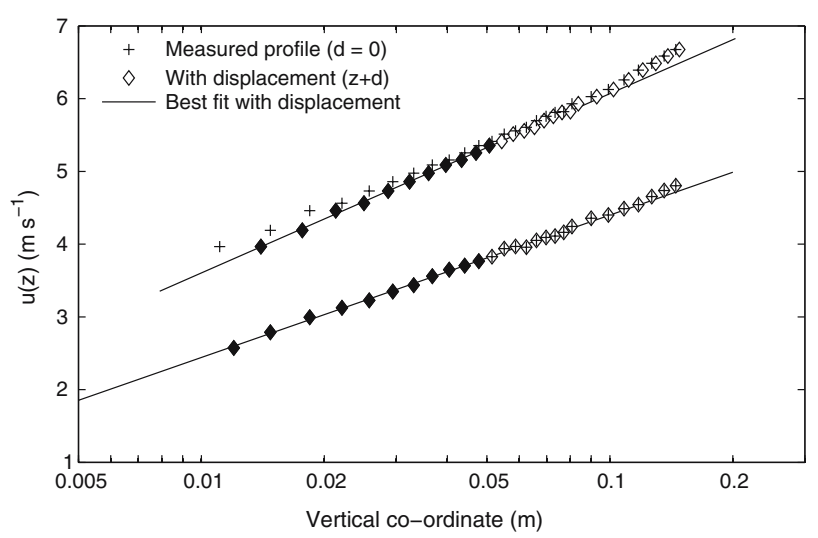

Fig. 5 Boundary-layer velocity profiles over snow. Data points in the assumed log region of the boundary layer are filled, and the resulting best fit line is shown

and fit. Adding the zero-plane displacement to the vertical co-ordinate results in a clear linear relationship between $u(z)$ and $\ln (z)$. By comparison, if $d=0, u_{*}=0.39 \mathrm{~m} \mathrm{~s}^{-1}$ and $z_{0}=0.17 \mathrm{~mm}$, and the fit $R^{2}$ decreases. The logarithmic region was assumed to extend to at least $z=50 \mathrm{~mm}$ at both speeds, and as can be seen from Figs. 4 and 5, may extend further.

The measured $u_{*}$ and $z_{0}$ compare well with the literature for snow, e.g., Clifton et al. (2006) or Andreas et al. (2005), where velocity profiles were used but the zero-plane displacement was neglected. This agreement is due to the low value of $d$ compared to the much greater height at which measurements are often made, implying that in Eq. $1, z+d \approx z$. While this might be a reasonable approach when using wind profile data to calculate $u_{*}$, it cannot be neglected for measurements near to the surface, or when modelling processes in the upper snow layers.

Measurements of velocity profiles over snow are inherently difficult, since they must be made at low $u_{*}$ to prevent drift, where the momentum transfer between drifting particles and the boundary layer could be confused with the influence of ventilation (Raupach 1991). However, low $u_{*}$ implies low free-stream velocities, and so measurement uncertainty is increased compared to higher $u_{*}$. Also, long duration experiments can result in metamorphosis of the snow on the surface, changing the roughness of the surface and also permeability. Because of these difficulties, literature data on the displacement height over natural snow surfaces is (as far as we are aware) completely lacking. In this respect, snow is not an ideal medium for experiments, and the rest of the experimental data presented in this paper will concentrate on measurements over foam surfaces.

\subsection{Foam Surfaces}

The measured $z_{0}$ at different $u_{*}$ for the different foam surfaces are plotted together with those from a solid, smooth floor in Fig. 6. Lines of constant $R e=u_{*} z_{0} / v$ are also shown, which give the relationship for a smooth floor and the boundary between the hydraulically rough and transitional regime from the literature. Literature data for the smooth wall lie in the region $0.105>R e_{*}>0.129$ (Schlichting and Gersten 2003; Bhaganagar et al. 2004), and the rough regime is $R e_{*}>70 e^{(-\kappa C)}$, where for a rough wall, $C \geq 8$ (Schlichting and Gersten 2003). 


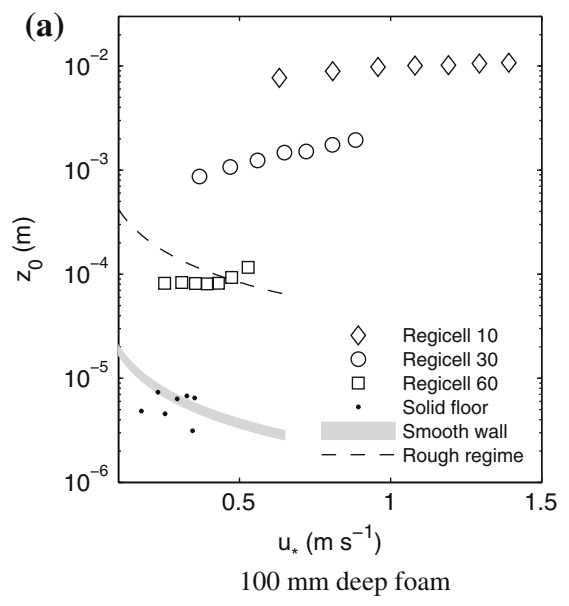

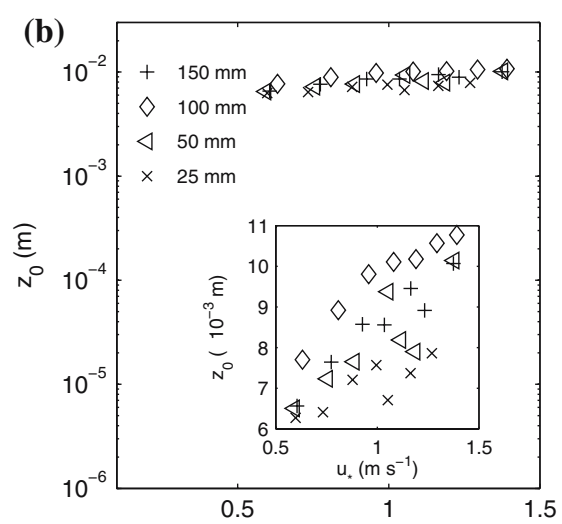

$\mathrm{u}_{\star}\left(\mathrm{m} \mathrm{s}^{-1}\right)$

Variable foam depth (Regicell 10). Note the inset figure showing the same data but with linear axes.

Fig. $6 z_{0}$ and $u_{*}$ for different foam surfaces. (a) $100 \mathrm{~mm}$ deep foams. Literature data for a smooth wall are shown by the region $0.105<R e_{*}<0.129$, where $R e_{*}=u_{*} z_{0} / v=e^{(-\kappa C)}$ (Schlichting and Gersten 2003; Bhaganagar et al. 2004; Raupach et al. 1991). The lower limit of the hydraulically rough regime is shown by the line $R e_{*}=2.6$. (b) Varying depths of Regicell 10

As was already shown in Fig. 4, solid, smooth floor measurements agree to better than $\pm 30 \%$ with the literature test cases. Replacing the solid smooth wall with a foam surface results in a clear increase in roughness length. The roughness length increases markedly with permeability; although all foam surfaces have no large-scale height variations, the roughness of the most permeable foam is two orders of magnitude higher than the least permeable foam. Zippe and Graf (1983) also observed a similar effect, noting that a permeable surface had a higher $z_{0}$ than an impermeable surface composed of the same material. The most permeable foam surfaces are well into the hydraulically rough regime, and the Regicell 60 is transitional. In the hydraulically rough regime, it has been shown that $z_{0}$ over an impermeable surface is independent of $u_{*}$ (Schlichting and Gersten 2003; Zagni and Smith 1976; Raupach et al. 1991). However, our data confirm the results of Zagni and Smith (1976), who observed that over a permeable surface, $z_{0}$ is a function of $u_{*}$. This is an important feature of flows over permeable surfaces, compared to solid rough surfaces. The gradient $\mathrm{d} z_{0} / \mathrm{d} u_{*}$ doubles from the Regicell 30 to the Regicell 10, suggesting that the increase in $z_{0}$ may be a result of increased permeability. The measured $z_{0}$ for the surfaces compares well with that seen for permeable natural materials; for example the Regicell 60 has a similar roughness length to the snow shown in Fig. 5, and $z_{0}$ of the Regicell 10 is similar to that for short grass of varying density, as summarised in Garratt (1994), Table A6, p. 290.

The depth of the most permeable foam was also changed from greater than $6 \ell_{\text {pore }}$ to greater than $38 \ell_{\text {pore }}$. The $u_{*}$ and $z_{0}$ calculated from the velocity profiles measured over the surface are plotted in Fig. 6b. Evidently, for this range of material depths, the depth does not greatly influence the roughness length. Any influence on the roughness length from the structure must therefore be dominated by momentum transfer processes in the upper pores of the medium.

As was noted in Sect. 2, the shear penetration would ideally be assessed by direct measurement in the pore space of the foam. As this was not possible, we use the displacement depth as indicators of momentum transfer. Results for all foams are plotted in Fig. 7. The zero-plane 

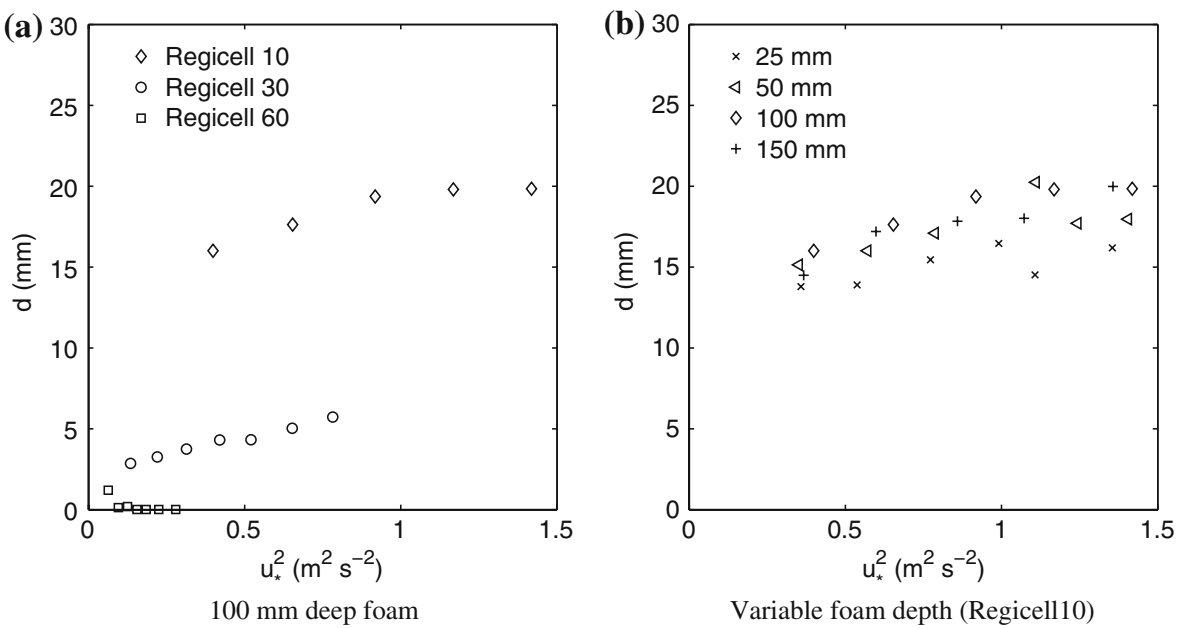

Fig. 7 Zero-plane displacement measured for foam surfaces

Table 3 Flow characteristics of foam experiments

\begin{tabular}{llllll}
\hline Case & $R_{k}$ & $z_{0} / \sqrt{k}$ & $z_{0} / d_{p}$ & $d / \sqrt{k}$ & $d / d_{p}$ \\
\hline Regicell 10 & $15.7-34.7$ & $19.3-26.9$ & $13.2-18.4$ & $40.0-52.7$ & $27.4-36.1$ \\
Regicell 30 & $4.5-10.8$ & $4.4-9.9$ & $4.0-8.9$ & $14.5-29.0$ & $13.0-26.1$ \\
Regicell 60 & $1.2-2.5$ & $1.1-1.5$ & $1.3-1.9$ & $0.0-16.0$ & $0.0-19.4$ \\
\hline
\end{tabular}

Data are shown for the $100 \mathrm{~mm}$ thick foam. Data show the range of $R e_{k}=u_{*} \sqrt{k} / v$, and the length scales $d$ and $z_{0}$ in Eq. 1 normalised by $\sqrt{k}$ and the equivalent particle diameter, $d_{p}$

displacement $d$ varies considerably between foams; $d$ is zero in many of the measurements over the least permeable foam, Regicell 60. By comparison, the displacement depth over the more permeable Regicell 10 and 30 is much larger, and increases with $u_{*}$. The displacement depth also decreased slightly as the depth of Regicell 10 was decreased, at similar $u_{*}$. At the maximum $u_{*}$ measured over the thinnest foam, the displacement depth was almost $70 \%$ of the depth of the foam. However, there is no suggestion of the zero-plane displacement asymptotically approaching the foam depth. The displacement depth was found to increase as the roughness length increased. This correlation between $z_{0}$ and $d$ also suggests that if snow permeability was sufficient to allow ventilation, drag from the snow microstructure might contribute to the aerodynamic roughness length.

Flow characteristics from the experiments are shown in Table3. The data include the permeability Reynolds number, and also the roughness length and zero-plane displacement, normalised by both $\sqrt{k}$ and the equivalent particle diameter of the foam, $d_{p}$. The results are within the range of results from direct numerical simulations by Breugem et al. (2006), which have the advantage of a much more highly resolved velocity profile than we have. Also, the results demonstrate an interesting effect of permeable walls compared to classical rough surfaces; the ratio $z_{0} / d_{p}$ is always greater than unity, and for the most permeable foam, approaches 20 . The same ratio over impermeable, densely packed rough surfaces is usually taken as 1/30 (Schlichting and Gersten 2003, p. 530), increasing as the surface roughness elements are moved apart. It is clear that there is scope for further investigation of this increase in $z_{0}$ with increasing permeability. 


\section{Discussion}

The data obtained for flat foam surfaces can be applied to snow by analogy. Measurements of the boundary layer over snow show that $z_{0}$ over natural surfaces is typically less than $1 \times 10^{-2} \mathrm{~m}$ (Andreas et al. 2005; Doorschot et al. 2004; Clifton et al. 2006). Wind speeds of $10 \mathrm{~m} \mathrm{~s}^{-1}$ are frequently measured at $10 \mathrm{~m}$ above snow surfaces in Antarctic regions (King and Turner 1997, p. 95) and in Greenland (Box et al. 2004). Taking $z_{0} \approx 1 \times 10^{-2} \mathrm{~m}$ implies a friction velocity of the order of $1 \mathrm{~m} \mathrm{~s}^{-1}$ in these environments. Depending on the surface, friction velocities above a threshold of around $0.3 \mathrm{~m} \mathrm{~s}^{-1}$ would usually be assumed to cause snow drift, although this threshold can increase or decrease depending on conditions (Clifton et al. 2006). However, drift would actually reduce the friction velocity at the snow surface to the drift threshold friction velocity (Raupach 1991). Snow surface permeability is typically less than $10 \times 10^{-9} \mathrm{~m}^{2}$, and often much lower (see Table 1). While values of up to $28 \times 10^{-9} \mathrm{~m}^{2}$ have been measured by Albert et al. (2000), this was found at a depth of $3 \mathrm{~m}$, and is not expected at the surface. By comparison, wind-packed surface snow at the same location was found to have a permeability of $10 \times 10^{-9} \mathrm{~m}^{2}$. The observations of Albert and Perron (2000) and Albert et al. (2000) suggest that the process of snow metamorphosis, resulting in rounded or faceted forms, reduces the SSA and increases the permeability. This is similar to that seen in the foams, where the foam with the lowest SSA has the highest permeability. This allows permeability gradients to develop both vertically within snow packs, and horizontally as local conditions change. Considering the range of $u_{*}$ found over snow surfaces, and the range of permeability of snow, the zero-plane displacement might realistically be expected to be similar to that of the Regicell 60 foam. From Fig. 7, this is usually zero. Even assuming that the permeability might rise to approximately that of the Regicell 30 (about twice the maximum values for snow surfaces that we have found in the literature), the zero-plane displacement is still less than $10 \mathrm{~mm}$ at $u_{*}<1 \mathrm{~m} \mathrm{~s}^{-1}$, which agrees with the limited observations we have from measurements over a snow surface.

Whilst a zero-plane displacement of the order of $10 \mathrm{~mm}$ appears large, this is the same order of magnitude as the height variations typically found in the surface of snow. This suggests that shear-driven ventilation is limited to the surface of the snow pack, most probably within the upper rugosities of the snow surface. It is possible that in some snow covers with exceptionally high permeability and very flat surfaces that net shear-driven flow may exist up to ten millimetres below the surface, but this requires permeability much higher than that found in the literature. The surface rugosities also cause local pressure variation and hence induce Darcy flow. The influence of the surface rugosities is local, and any resulting flow might be found as well as, or instead of, flow caused by downwards momentum transfer. These two effects are sketched in Fig. 8.

As the surface roughness of snow induces local ventilation, so other features can also cause localised pressure-driven flow within the snow pack. This would include such sub metre-scale features as sastrugii, larger features like dunes, and also the local surface relief.

Fig. 8 Velocity profiles over a snow surface. Profiles are shown without a mean pressure gradient in the streamwise direction $(d P / d x=0)$. Dashed lines indicate possible flow paths within the snow. Flow regimes I and II correspond to those shown in Fig. 1

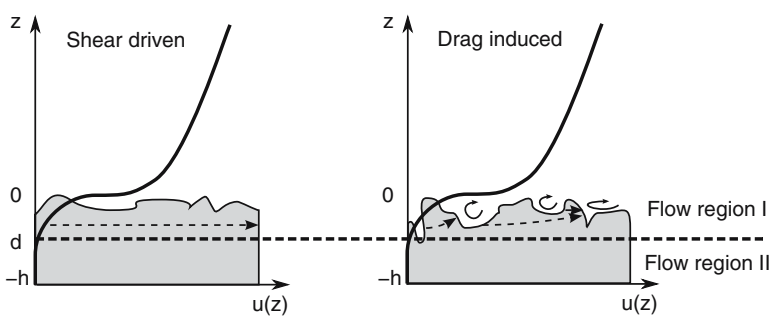


However, this kind of drag-induced ventilation is due to fundamentally different physics to shear-driven flow, and as was shown in Fig. 1, results in different flow characteristics. As a result, topology influenced, pressure-driven flow should be considered (and modelled) separately to any shear-driven flow. Since for simple one-dimensional simulations of the snow cover these processes cannot be separated, and natural snow surfaces often have a higher roughness than the examples in the wind tunnel, parameterisation of enhanced heat and vapour transport in the uppermost snow layers may still be necessary and are supported by the fact that improved snow cover simulations result (Lehning et al. 2002).

\section{Conclusions}

We have presented a method for assessing shear-driven ventilation of snow packs using mean velocity profiles measured in the boundary layer immediately above the surface. We suggest that the zero-plane displacement can be used as an indicator of shear-driven ventilation, and suggest that as the displacement depth tends to zero, so does the downwards momentum transfer into the snow pack. We suggest that future efforts should be targeted towards direct measurement of the velocities in the upper pores of the media, which are accessible from above. This would allow direct evaluation of ventilation, rather than extrapolation from the velocity profile.

First measurements over snow of unknown permeability show a $3 \mathrm{~mm}$ displacement depth in one case, but difficulties in making reliable measurements over snow preclude an exhaustive test series. Instead, foams with a range of permeabilities were used as analogous materials, allowing the influence of the material structure on the velocity profile to be investigated.

Although the foam surfaces are flat, their roughness length increased as the foam permeability changed. Roughness lengths increased from $1 \times 10^{-4}$ to $1 \times 10^{-2} \mathrm{~m}$ as the permeability was changed from $6 \times 10^{-9}$ to $160 \times 10^{-9} \mathrm{~m}^{2}$. The roughness length was also found to increase slightly with $u_{*}$ when the permeability was kept constant. The zero-plane displacement was found to be generally less than $1 \mathrm{~mm}$ for the foam with the lowest permeability, which is similar to that of snow. By comparison, the displacement depth of the most permeable foam was $15-20 \mathrm{~mm}$, and increased with $u_{*}$. These results are within the range of values reported by Breugem et al. (2006) from numerical simulations of flow over flat permeable walls at similar Reynolds numbers.

The pressure gradient in the test section of the wind tunnel was zero, and any mean flow in the streamwise direction into the medium was therefore caused by downwards momentum transport. Our results suggest that at low permeabilities, such as those found in snow, the zero-plane displacement is of similar size to the surface geometrical roughness, and thus shear-driven ventilation is confined to the surface of a snow cover. By implication, turbulent fluxes of scalars (for example sensible and latent heat and tracer substances) are also confined to that region. Below this region, any scalar transfer is limited to molecular diffusion processes and pressure-gradient driven flows. The role of inactive turbulence and the contribution of these processes to heat fluxes and associated snow metamorphosis remain an interesting and valid research topic.

Acknowledgements This work was partially financed by the Swiss National Foundation. T. Exner, R. Grant, D. Ambühl, V. Smith, A. Craig and the SLF Workshop were invaluable for help preparing the wind tunnel and the techniques we applied. We thank T. Neumann and Z. Courville for measuring the foam permeability. 


\section{References}

Albert M, Perron F (2000) Ice layer and surface crust permeabilty in a seasonal snow pack. Hydrol Process $14: 3207-3214$

Albert M, Schultz E (2002) Snow and firn properties and air-snow transport processes at Summit, Greenland. Atmos Environ 36:2789-2797

Albert M, Schultz E, Perron F (2000) Snow and firn permeability at Siple Dome, Antarctica. Ann Glaciol 31:353-356

Albert M, Grannas A, Bottenhiem J, Shepson P, Perron F (2002) Processes and properties of snow-air transfer in the high Arctic with application to interstitial ozone at Alert, Canada. Atmos Environ 36:2779-2787

Andreas E, Jordan R, Makshtas A (2005) Parameterizing turbulent exchange over sea ice: the Ice Station Weddell results. Boundary-Layer Meteorol 114:439-460

Bhaganagar K, Kim J, Coleman G (2004) Effect of roughness on wall-bounded turbulence. Flow, Turbul Combust 72:463-492

Box J, Bromwhich D, Bai LS (2004) Greenland ice sheet surface mass balance for 1991-2000: application of Polar MM5 mesoscale model and in-situ data. J Geophys Res 109(D16). Doi 10.1029/2003JD004451

Breugem W, Boersma B, Uittenbogaard R (2006) The influence of wall permeability on turbulent channel flow. J Fluid Mech 562(1):35-72

Clauser F (1954) Turbulent boundary layers in adverse pressure gradients. J Aeronaut Sci 21:91-105

Clifton A, Rüedi JD, Lehning M (2006) Snow saltation threshold measurements in a drifting-snow wind tunnel. J Glaciol 52(179):585-596

Colbeck S (1989) Air movement in snow due to windpumping. J Glaciol 35(120):209-213

Doorschot J, Lehning M, Vrouwe A (2004) Field measurements of snow drift and mass fluxes and comparison with model simulations. Boundary-Layer Meteorol 113:347-368

Garratt J R (1994) The atmospheric boundary layer. Cambridge University Press, U.K., 316 pp

Jackson P (1981) On the displacement height in the logarithmic velocity profile. J Fluid Mech 111:15-25

King JC, Turner J (1997) Antarctic meteorology and climatology. Cambridge University Press, U.K., 425 pp

Lehning M, Bartelt P, Brown B, Fierz C (2002) A physical SNOWPACK model for the Swiss avalanche warning part III: meteorological forcing, thin layer formation and evaluation. Cold Reg Sci Technol 35(3):169-184

Manes C (2006) Turbulent open channel flow over and within rough and permeable beds. PhD thesis, University of Aberdeen

McKeon B, Li J, Jiang W, Morrison J, Smiths A (2003) Pitot probe corrections in fully developed turbulent pipe flow. Meas Sci Technol 14:1449-1458

Moffat R (1982) Contributions to the theory of single-sample uncertainty analysis. J Fluids Eng 104:250-260

Nikora V, Koll K, McLean S, Dittrich A, Aberle J (2002) Zero-plane displacement for rough-bed open-channel flows. In: Bousmar D (ed) River Flow 2002 : proceedings of the International Conference on Fluvial Hydraulics, Louvain-la-Neuve, Belgium, 4-6 September 2002, International Conference on Fluvial Hydraulics

Raupach M (1991) Saltation layers, vegetation canopies and roughness lengths. Acta Mechanica (Suppl) (1):83-96

Raupach MR, Antonia R, Rajagopalan S (1991) Rough-wall turbulent boundary layers. Appl Mech Rev 44(1): $1-24$

Schlichting H, Gersten K (2003) Boundary layer theory, 8th edn. Springer Verlag, 799 pp

Schneebeli M, Sokratov S (2004) Tomography of temperature gradient metamorphism of snow and associated changes in heat conductivity. Hydrol Process 18:3655-3665

Sokratov S, Sato A (2000) Wind propagation to snow observed in laboratory. Ann Glaciol (31):427-433

Sokratov S, Sato A (2001) The effect of wind on the snow cover. Ann. Glaciol (32)

Sommerfeld R, Rocchio J (1993) Permeability measurements on new and equitemperature snow. Water Resour Res 29(8):2485-2490

Sturm M, Johnson B (1991) Natural convection in the subarctic snow cover. J Geophy Res 96(B7):11,65711,671

Thom A (1971) Momentum absorption by vegetation. Quart J Roy Meteorol Soc 97:414-428

Waddington E, Cunningham J (1996) The effect of snow ventilation on chemical concentrations. In: Wolff EW, Bales RG (eds) Chemical exchange between the atmosphere and polar snow, NATO ASI series, vol. 143, pp 403-451

Zagni A, Smith K (1976) Channel flow over permeable beds of graded spheres. J Hydraulics Division ASCE 102:207-22

Zippe H, Graf W (1983) Turbulent boundary-layer flow over permeable and non-permeable rough surfaces. J Hydraulic Res 21(1):51-65 